\title{
ON THE REPRESENTATION OF $\sigma$-COMPLETE BOOLEAN ALGEBRAS
}

\author{
L. H. LOOMIS
}

A $\sigma$-complete Boolean algebra is a Boolean algebra in which for every sequence of elements $a_{i}, i=1, \cdots$, there is an element $U_{1}^{\infty} a_{n}$, the countable union of the $a_{i}$, such that $a_{i} \subseteq \bigcup_{1}^{\infty} a_{n}$ for every $i$, and such that if $a_{i} \subseteq x$ for every $i$ then $\cup_{1}^{\infty} a_{n} \subseteq x$. The dual operation, countable intersection, can be introduced through complementation, and the distributive law $a \cap \bigcup_{1}^{\infty} a_{n}=\bigcup_{1}^{\infty}\left(a \cap a_{n}\right)$ and its dual can be proved (see $\left[3\right.$, p. 93]). ${ }^{1}$ Certain types of Boolean algebras have representations as algebras of point sets, the representation preserving all the operations of the algebra. Among these are ordinary Boolean algebras with no further operations (Stone $[1$, p. 106]) and complete Boolean algebras for which very general operations and distributive laws are assumed (Tarski [2, pp. 197-198]). On the other hand it is well known that a $\sigma$-complete Boolean algebra has in general no such representation. For example, the quotient of the algebra of Lebesgue measurable subsets of $[0,1]$ modulo the ideal of sets of measure zero is a $\sigma$-complete Boolean algebra which is not $\sigma$-isomorphic to any $\sigma$-complete Boolean algebra of point sets. The following theorem, which we shall prove in this note, shows that this example illustrates the general situation.

THEOREM. Every $\sigma$-complete Boolean algebra is $\sigma$-isomorphic to a $\sigma$-complete Boolean algebra of point sets modulo a $\sigma$-ideal in that algebra.

In particular, every abstract measure algebra can be considered as an algebra of point sets modulo sets of measure zero. Bischof [4] has recently obtained a proof of this special case of the theorem, but his proof leans heavily on the existence of a measure and does not generalize.

If we are given a representation of an abstract collection $R$ of objects onto a family $F$ of sets, then each point $p$ of the representation space determines a subfamily of sets $F_{p}$ and hence a subcollection $R_{p}$ of $R$, namely, those objects in $R$ whose image sets in $F$ contain $p$. This suggests the way to define points in attempting to build up a representation. A point will be a certain kind of subset of $R$, the image $\mathfrak{a}$ of $a$ will be the set of points containing $a$, and the representation $\Re$ of $R$

Received by the editors February 12, 1947.

1 Numbers in brackets refer to the bibliography at the end of the paper. 
will be the collection of such sets $\mathfrak{a}$. If $R$ is closed under a complementation operation and the representation is to preserve complements, then clearly a point must be a "selection subset" of $R$, which for each $a \in R$ contains $a$ or $a^{\prime}$ but not both. If $R$ is a Boolean algebra and the representation is to be an isomorphism with respect to Boolean operations, then a point $P$ must be a selection subset and must have the further properties that (1) if $a \in R$ and $b \in P$ then $a \cup b \in P$, and (2) if $a_{i} \in P, i=1, \cdots, n$, then $\bigcap_{i}^{n} a_{i}$ is in $P$ and is not null. Such a subset of $R$ is called a dual prime ideal. Stone's theorem asserts that the correspondence $a \rightarrow \mathfrak{a}$, where $\mathfrak{a}$ is the set of dual prime ideals containing $a$, is actually an isomorphism. If $R$ is a $\sigma$-complete Boolean algebra and the representation is to be a $\sigma$-isomorphism, then the point $P$ must be not only a dual prime ideal, but must also have the stronger multiplicative property that if $a_{i} \in P, i=1, \cdots, \infty$, then $\cap_{1}^{\infty} a_{i}$ is in $P$ and is not null. Such a dual ideal could be called a dual prime $\sigma$-ideal. It can easily be shown that the correspondence $a \rightarrow \mathfrak{a}$ is a $\sigma$-isomorphism if and only if the set $\mathfrak{a}$ of dual prime $\sigma$-ideals containing $a$ is nonvoid for every non-null $a$ in $R$. In general there are not enough dual prime $\sigma$-ideals. In fact, the above mentioned algebra of measurable subsets of $[0,1]$ modulo sets of measure 0 has no dual prime $\sigma$-ideals. For let $a_{i}$ be the set of numbers in $[0,1]$ which in dyadic representation have 0 at the $i$ th place. Then $m\left(a_{i}\right)=m\left(a_{i}^{\prime}\right)=1 / 2$, and the measure of the product of any $n$ such sets or their complements is at most $2^{-n}$. Thus any dual prime $\sigma$-ideal would have to contain an infinite product of measure 0 and consequently, in the quotient space, a null product, which contradicts the definition of a dual prime $\sigma$-ideal. This proves the assertions we have made about this example, and it also shows that in defining points for our representation we cannot use the stringent requirements of dual prime $\sigma$-ideals.

We shall, in fact, go back to our weakest definition and take a point to be simply a selection subset with respect to complementation. We repeat that the set $\mathfrak{a}$ corresponding to the element $a$ is the set of all points containing $a$, and that $\Re$ is the collection of such sets $a$. The correspondence $a \rightarrow \mathfrak{a}$ clearly preserves complementation. Let $\mathfrak{B}(\Re)$ be the $\sigma$-complete Boolean algebra (Borel field) of point sets generated by $\Re$, that is, the smallest family of sets including $\Re$ and closed under the operations of complementation and countable union. Let $\mathfrak{N}$ be the family of sets of $\mathfrak{B}(\Re)$ of the form $\bigcap_{i}^{n} \mathfrak{a}_{i}$ if $\bigcap_{i}^{n} a_{i}=0$ or $\bigcap_{1}^{\infty} \mathfrak{a}_{i}$ if $\bigcap_{1}^{\infty} a_{i}=0$. Let $\Im(\mathfrak{R})$ be the $\sigma$-ideal generated by $\mathfrak{R}$, that is, the smallest family of sets including $\mathfrak{R}$ and closed under the operations of countable union and of intersection with any set of $\mathfrak{B}(\Re)$. Let $\bar{\Re}$ be the quotient $\sigma$-complete Boolean algebra $\mathfrak{B}(\mathfrak{R}) / \mathfrak{\Im}(\mathfrak{R})$ and let $\overline{\mathfrak{a}}$ be the ele- 
ment (coset) of $\bar{\Re}$ containing $\mathfrak{a}$. The theorem can be stated more exactly as follows.

THEOREM. The $\sigma$-complete Boolean algebras $R$ and $\bar{\Re}$ are $\sigma$-isomorphic under the correspondence $a \rightarrow \bar{a}$.

First, the correspondence $a \rightarrow \bar{a}$ is a $\sigma$-homomorphism onto the whole of $\bar{\Re}$. For if $a=\bigcup_{1}^{\infty} a_{n}$ then $a \cap \bigcap_{1}^{\infty} a_{n}^{\prime}=0$ and $a^{\prime} \cap a_{i}=0$ for every $i$. Therefore, by the definition of $\mathfrak{N}, \mathfrak{a} \cap \cap_{1}^{\infty} \mathfrak{a}_{n}^{\prime} \in \mathfrak{N}$ and $\mathfrak{a}^{\prime} \cap \mathfrak{a}_{i} \in \mathfrak{N}$ for every $i$, so that $\overline{\mathfrak{a}}=\bigcup_{1}^{\infty} \overline{\mathfrak{a}}_{n}$, which proves the correspondence to be a $\sigma$-homomorphism. The images $\overline{\mathfrak{a}}$ of the elements of $R$ thus form a $\sigma$-complete Boolean algebra and hence cover the whole of $\bar{\Re}$ (which is generated by these images). That this $\sigma$-homomorphism onto $\bar{\Re}$ is a $\sigma$-isomorphism is the obvious content of the following lemma, the proof of which depends ultimately upon an application of the diagonal process.

LEMmA. If $\overline{\mathfrak{a}}=0$ (that $i s, a \in \Im(\mathfrak{N})$ ) then $a=0$.

Any element of $\mathfrak{I}(\mathfrak{N})$ is included in a countable sum of elements of $\mathfrak{N}$, for the elements of $\mathfrak{\Im}(\mathfrak{N})$ having this property clearly form a $\sigma$-ideal including $\mathfrak{R}$. Thus, to prove the lemma it is sufficient to prove that

$$
\text { if } a \subseteq \bigcup_{n=1}^{\infty} \bigcap_{m=1}^{\infty} \mathfrak{a}_{m}^{n} \text { then } a \subseteq \bigcup_{n=1}^{\infty} \bigcap_{m=1}^{\infty} a_{m}^{n}
$$

Because of the more intuitive distributive law involved we shall prove the complementary form

$$
\text { if } \bigcap_{n=1}^{\infty} \bigcup_{m=1}^{\infty} \mathfrak{a}_{m}^{n} \subseteq \mathfrak{a} \quad \text { then } \bigcap_{n=1}^{\infty} \bigcup_{m=1}^{\infty} a_{m}^{n} \subseteq a \text {. }
$$

The hypothesis of (2) implies that

$$
\bigcap_{n=1}^{\infty} \mathfrak{a}_{m(n)}^{n} \subseteq \mathfrak{a} \quad \text { for every function } m(n) .
$$

This can occur only if (i) $a$ occurs in the sequence $a_{m(n)}^{n}$, or if (ii) the sequence contains a complementary pair. For otherwise we can form a point $P$ containing all the elements $a_{m(n)}^{n}$ and also containing $a^{\prime}$, invalidating the inclusion (3). But the conditions (i) or (ii) imply the conclusion of (2). For suppose on the contrary that

$$
a^{\prime} \cap\left(\bigcap_{n=1}^{\infty} \bigcup_{m=1}^{\infty} a_{m}^{n}\right)=b \not 00 .
$$


Since $b \subseteq \bigcup_{m=1}^{\infty} a_{m}^{n}$ for every $n$, we can find an index $m(1)$ such that $b \cap a_{m(1)}^{1} \neq 0$, an index $m(2)$ such that $\left(b \cap a_{m(1)}^{1}\right) \cap a_{m(2)}^{2} \neq 0$, and, inductively, indices $m(i)$ such that $b \cap \bigcap_{1}^{n} a_{m(i)}^{i} \neq 0$. The sequence $a_{m(i)}^{i}$ so chosen cannot have the property (ii), and since $b \subseteq a^{\prime}$ it cannot have the property (i). The hypothesis (4) thus leads to a contradiction and (2) has been established.

\section{BIBLIOGRAPHY}

1. M. H. Stone, The theory of representations for Boolean algebras, Trans. Amer. Math. Soc. vol. 40 (1936) pp. 37-111.

2. A. Tarski, Zur Grundlegung der Boole'schen Algebra I, Fund. Math. vol. 24 (1935) pp. 177-198.

3. G. Birkhoff, Lattice theory, Amer. Math. Soc. Colloquium Publications, vol. 25,1940 .

4. A. Bischof, Beitrage zur CarathEodoryschen Algebraischen des Integralsbegriffs, Schriften des Matematischen Instituts und des Instituts für angewandte Mathematik der Universität Berlin, 1941, pp. 238-263.

HARVARD UNIVERSITY 